\title{
Optimised biomolecular extraction for metagenomic analysis of microbial biofilms from high-mountain streams
}

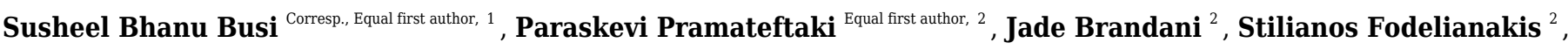 \\ Hannes Peter ${ }^{2}$, Rashi Halder ${ }^{1}$, Paul Wilmes ${ }^{1}$, Tom J. Battin ${ }^{2}$ \\ ${ }^{1}$ Systems Ecology Research Group, Luxembourg Centre for Systems Biomedicine, University of Luxembourg, Esch-sur-Alzette, Luxembourg \\ 2 Stream Biofilm and Ecosystems Research group, École Polytechnique Federale de Lausanne, Lausanne, Switzerland \\ Corresponding Author: Susheel Bhanu Busi \\ Email address: susheelbhanu@gmail.com
}

Glacier-fed streams (GFS) are harsh ecosystems dominated by microbial life organized in benthic biofilms, yet the biodiversity and ecosystem functions provided by these communities remain under-appreciated. To better understand the microbial processes and communities contributing to GFS ecosystems, it is necessary to leverage high throughput sequencing. Low biomass and high inorganic particle load in GFS sediment samples may affect nucleic acid extraction efficiency using extraction methods tailored to other extreme environments such as deep-sea sediments. Here, we benchmarked the utility and efficacy of four extraction protocols, including an up-scaled phenol-chloroform protocol. We found that established protocols for comparable sample types consistently failed to yield sufficient high-quality DNA, delineating the extreme character of GFS. The methods differed in the success of downstream applications such as library preparation and sequencing. An adapted phenol-chloroform-based extraction method resulted in higher yields and better recovered the expected taxonomic profile and abundance of reconstructed genomes when compared to commercially-available methods. Affordable and straight-forward, this method consistently recapitulated the abundance and genomes of a "mock" community, including eukaryotes. Moreover, by increasing the amount of input sediment, the protocol is readily adjustable to the microbial load of the processed samples without compromising protocol efficiency. Our study provides a first systematic and extensive analysis of the different options for extraction of nucleic acids from glacierfed streams for high-throughput sequencing applications, which may be applied to other extreme environments. 
1 Optimised biomolecular extraction for metagenomic analysis of microbial biofilms from 2 high-mountain streams

3 Susheel Bhanu Busi 1,\#,*, Paraskevi Pramateftaki²,\#, Jade Brandani², Stilianos Fodelianakis ${ }^{2}$, 4 Hannes Peter ${ }^{2}$, Rashi Halder ${ }^{1}$, Paul Wilmes ${ }^{1}$ and Tom J. Battin ${ }^{2}$

5

$6{ }^{1}$ Systems Ecology Research Group, Luxembourg Centre for Systems Biomedicine, University of 7 Luxembourg, Esch-sur-Alzette, Luxembourg

$8{ }^{2}$ Stream Biofilm and Ecosystems Research group, Ecole Polytechnique Fédérale de Lausanne, 9 Lausanne, Switzerland

10

${ }^{\#}$ Co-first authors

12

*Corresponding author: Susheel Bhanu Busi

14 Address: 7 avenue des hauts-fourneaux, BioTech-I, Luxembourg Centre for Systems Biomedicine, 15 University of Luxembourg, Esch-sur-Alzette, L-4362 Luxembourg

Email: susheel.busi@uni.lu

\section{Abstract}

Glacier-fed streams (GFS) are harsh ecosystems dominated by microbial life organized in benthic biofilms, yet the biodiversity and ecosystem functions provided by these communities remain under-appreciated. To better understand the microbial processes and communities contributing to GFS ecosystems, it is necessary to leverage high throughput sequencing. Low biomass and high inorganic particle load in GFS sediment samples may affect nucleic acid extraction efficiency using extraction methods tailored to other extreme environments such as deep-sea sediments. Here, we benchmarked the utility and efficacy of four extraction protocols, including an up-scaled phenol-chloroform protocol. We found that established protocols for comparable sample types consistently failed to yield sufficient high-quality DNA, delineating the extreme character of GFS. The methods differed in the success of downstream applications such as library preparation and sequencing. An adapted phenol-chloroform-based extraction method resulted in higher yields and better recovered the expected taxonomic profile and abundance of reconstructed genomes when 
32 consistently recapitulated the abundance and genomes of a "mock" community, including 33 eukaryotes. Moreover, by increasing the amount of input sediment, the protocol is readily 34 adjustable to the microbial load of the processed samples without compromising protocol 35 efficiency. Our study provides a first systematic and extensive analysis of the different options for 36 extraction of nucleic acids from glacier-fed streams for high-throughput sequencing applications, 37 which may be applied to other extreme environments.

\section{Introduction}

40 The advent of high-throughput sequencing technologies has brought hitherto inconceivable 41 capacities to characterize the microbial ecology of both well-studied (Jansson and Hofmockel 2018; Nielsen and Ji 2015) and under-explored environments (Hotaling, Hood, and Hamilton 2017; Milner et al. 2017). Among the latter include high-mountain and particularly glacier-fed streams (Milner et al. 2017) and the microbial biofilms that colonize their beds (Battin et al. 2016). Today, these streams are changing at an unprecedented pace owing to climate change and the thereby shrinking glaciers, and yet little is known of their microbial diversity (Wilhelm et al. 2013, Milner et al. 2017). Glacier-fed stream (GFS) sediments are extreme habitats characterized by low microbial cell abundance and activities but very high loads of fine mineral particles (Wilhelm et al. 2013; Godone 2017; Peter and Sommaruga 2017; Chanudet and Filella 2008; Bogen 1988). In order to understand the diversity and composition of these microbial communities, including both eukaryotes and prokaryotes, and the role that they play, it is essential to extract nucleic acids in sufficient quantity and quality from often complex environmental matrices. After extracting the nucleic acids, downstream applications including molecular biology methods such as PCR and next-generation sequencing of amplicons or metagenomes allow for the compositional, functional and phylogenetic characterization of microbial populations and the communities that they form (Roume et al. 2013).

While there is no lack of protocols and literature pertaining to the extraction of nucleic acids from a wide variety of environments (Roume et al. 2013; Miller et al. 1999; Xin and Chen 2012; Porebski, Bailey, and Baum 1997; Zhou, Bruns, and Tiedje 1996), few reports dwell on the utility of these methods for biomolecular extractions from sedimentary samples with very low cell abundance as typical for GFS (Wilhelm et al. 2013; Ren, Gao, and Elser 2017). In 2015, Lever et 
63 al. elaborately described diverse factors and components that need to be considered for efficient 64 nucleic acid extractions (Lever et al. 2015). These include, but are not limited to key steps like cell 65 lysis, removal of impurities and inhibitors and of critical additives like carrier DNA molecules to 66 enhance aggregation and thus precipitation of DNA in case of very low concentrations. Since the 67 first extraction of DNA by Swiss medical doctor Friedrich Miescher in 1869 (Dahm 2008), 68 biomolecule extractions have shifted from those performed with solutions prepared primarily in 69 the laboratory (Sambrook and Russell 2006; Miller et al. 1999) to using commercially-available 70 kits. These ready-made options are designed to avoid the use of volatile and toxic chemicals such 71 as phenol and chloroform, and are tailored to various environments including blood, faecal 72 material, plant and soils (Claassen et al. 2013; Psifidi et al. 2015; Smith, Diggle, and Clarke 2003; 73 Vishnivetskaya et al. 2014). While studies have concentrated on nucleic acid extraction from 74 glacial ice cores (Dancer, Shears, and Platt 1997) or surface snow (Pei-Ying et al. 2012), none has 75 76 demonstrated their utility for GFS sediments. Together with low cell abundance (Wilhelm et al. 2014; Lever et al. 2015), the complex mineral matrices in GFS (Peter and Sommaruga 2017)- a consequence of the erosion activity of glaciers (Bogen 1988)- may affect nucleic acid extraction efficiency (Lever et al. 2015). As we attempt to better understand how nature works at its limits through the study of extreme environments, non-commercial approaches (Mukhopadhyay and Roth 1993)and methodologies (Lever et al. 2015), need to be revisited and optimized.

In recent years, several research groups (Besemer et al. 2012; Ren et al. 2017; Ren, Gao, and Elser 2017; Dancer, Shears, and Platt 1997) have successfully used kit-based methods for DNA extraction and subsequent 16S ribosomal RNA gene amplicon sequencing on GFS samples. However, the requirements for whole genome shotgun sequencing currently include at least $50 \mathrm{ng}$ of input DNA to minimize bias due to PCR reactions during library preparation (Kebschull and 87 Zador 2015; Bowers et al. 2015; Thomas, Gilbert, and Meyer 2012; Chafee, Maignien, and Simmons 2015; Peng et al. 2020). Here, we address the utility and efficiency of the "gold" standard phenol-chloroform extraction (Dairawan and Shetty 2020), and three alternative methodologies to identify the process(es) that yield not only the highest quantity but also quality of DNA, from GFS sediments. Our goal was to address whether the phenol-chloroform method yielded the expected diversity and taxonomic profiles when extracting GFS sediments, while also enabling 93 reconstruction of metagenome-assembled genomes. Simultaneously, we wanted to validate the 
94 utility of this method for the extraction of nucleic acids from both pro- and eu- karyotic sources. 95 Overall, our findings provide a framework for the extraction of nucleic acids such as DNA for whole genome shotgun sequencing from GFS sediments, whilst highlighting the potential variability introduced due to the isolation method employed.

\section{Methods}

\section{Sample origin \& collection}

DNA extraction protocols were benchmarked using three different GFS sediments from the Swiss Alps: Corbassière (CBS, collection date: 13.11.2018), 2444 meters above sea level (m a.s.1) and Val Ferret (FE), $1995 \mathrm{~m}$ a.s.1 at the glacier snout (up site, FEU, collection date: 23.10.2018) and one kilometer further downstream (down site, FED, collection date: 24.01.2019). Sampling was always performed later in the morning, before noon. Sediments $(0.25$ to $3.15 \mathrm{~mm})$ were collected using two flame-sterilized metal sieves with a mesh size of $3.15 \mathrm{~mm}$ and $0.250 \mathrm{~mm}$ respectively. CBS differs from FEU and FED in terms of bedrock geology, with clastic sedimentary limestone dominating the catchment of CBS and Brecchia of gneiss dominating in FEU and FED. Sediments generally contain more organic material further downstream from the glacier, which may inhibit DNA extraction. Sediments $(0.25$ to $3.15 \mathrm{~mm})$ were collected using flame-sterilized sampling equipment. Wet sediments were transferred into $10 \mathrm{ml}$ sterile, DNA/DNase-free tubes and immediately flash-frozen in liquid nitrogen in the field. Samples were transferred to the laboratory and kept at $-80{ }^{\circ} \mathrm{C}$ until analysis. All necessary measures were taken to ensure contamination-free sampling.

\section{DNA extraction methods}

Four different DNA extraction methods were applied to the samples. The key characteristics of the different methods are summarized in Table 1. Method-1, -2 and -4 were manual protocols differing primarily in the lysis step (bead-beating and lysis buffer composition; (Roume et al. 2013; Lever et al. 2015; Sambrook and Russell 2006) while method-3 was a modified protocol of the DNeasy PowerMax Soil Kit (Cat.No. 12988-10) provided by Qiagen (based on communication exchanged with the manufacturer). Due to the very low microbial abundance, additional precautions were taken to establish contamination-free conditions, including daily decontamination of 
124 equipment/areas with bleach, using DNA/DNase-free glassware and plasticware, reagents and 125 chemicals. Additionally, "germ-free" sediment is not a viable option and is hard to remove any 126 and all microorganisms from sediments. So, extraction blanks, i.e. tubes without any sample, were 127 used as controls, which underwent the same extraction protocols along with the other samples. 128 Post-DNA recovery, we assessed whether any of the eluted samples had DNA via both NanoDrop and Qubit, and found them to be below detectable levels. Additionally, the PCR reactions during library preparation did not yield any product confirming serving as a contamination-check. The input weight of sediment ranged from $0.5-5 \mathrm{~g}$ and are described in the respective protocols.

Method-1 was based on a previously established method (Griffiths et al. 2000). Introduced modifications concerned primarily the step of mechanical lysis and DNA precipitation that was rendered more stringent to improve the recovery of small amounts of DNA. Sample cell lysis was achieved by adding $0.5 \mathrm{~g}$ of sediment into a lysing matrix $\mathrm{E}$ tube with beads of variable diameter provided by the manufacturer (MP Biomedicals, SKU 116914050), $500 \mu$ CTAB buffer (5\% $\mathrm{CTAB}, 120 \mathrm{mM} \mathrm{KPO}_{4}, \mathrm{pH}$ 8.0) and $500 \mu \mathrm{l}$ of phenol/chloroform/isoamyl alcohol (ratio 25:24:1). Samples were loaded on a Precellys beater for $45 \mathrm{~s}$ at $5.500 \mathrm{r} / \mathrm{s}$. DNA was extracted once more with chloroform/isoamyl alcohol (24:1) and precipitated with 2 vol PEG-6000, $15 \mu \mathrm{g} / \mathrm{ml}$ linear polyacrylamide (LPA) and $2 \mathrm{~h}$ incubation on ice (Supplementary material).

Method-2 was an adaptation to alpine stream sediments of the modular method for DNA extraction previously published (Lever et al. 2015). The appropriate modules of the method, based on the nature of our samples, were put together in our protocol without further modification. Samples were prepared by mixing $5 \mathrm{~g}$ of sediment, $10-20 \%$ of $0.1 \mathrm{~mm}$ zirconium beads and $1 \mathrm{ml}$ of $100 \mathrm{mM}$ dNTP solution. Cell lysis was achieved with $5 \mathrm{ml}$ lysis buffer $(30 \mathrm{mM}$ Tris-HCl, $30 \mathrm{mM}$ EDTA, $1 \%$ Triton $\mathrm{X}-100,800 \mathrm{mM}$ guanidium hydrochloride, $\mathrm{pH} 10.0$ ) and incubation at $50^{\circ} \mathrm{C}$ for $1 \mathrm{~h}$ with gentle agitation (Hybridization oven, Labnet Problot L6). The supernatant was extracted once with chloroform/isoamyl alcohol (24:1) and DNA was precipitated with $10 \mu \mathrm{g} / \mathrm{ml}$ LPA, 0.2 vol $0.5 \mathrm{M}$ $\mathrm{NaCl}, 2.5 \mathrm{vol}$ ethanol and $2 \mathrm{~h}$ incubation at RT in the dark (Supplementary material). The input weight of $5 \mathrm{~g}$ sediment was a modification from previously established protocol and the subsequent reagent volumes were adjusted accordingly. 
155 Method-3 has been previously applied successfully on sand and clay soils (Hale and Crowley 156 2015). In this protocol, the standard lysis capacity of the DNeasy PowerMax Soil Kit (Qiagen, Cat. 157 No. 112988-10) was modified and enhanced by the addition of phenol:chloroform:isoamyl alcohol 158 along with PowerBeads (kit provided) and C1 solution to $5 \mathrm{~g}$ of sediment. Then, the manufacturer159 suggested sequence of treatments and rinses with the standard buffers of the kit were followed to 160 reach elution of extracted DNA from silica columns with $6 \mathrm{ml}$ of elution buffer. Further 161 concentration of extracted DNA was carried out with the addition of $240 \mu \mathrm{l} 5 \mathrm{M} \mathrm{NaCl}, 2.5 \mathrm{vol}$ 162 ethanol and $10 \mu \mathrm{g} / \mathrm{ml}$ LPA (Supplementary material). LPA was an additional modification to the 163 original protocol for improved DNA recovery.

165 166

167 168

Method-4 involved chemical and enzymatic treatment of samples according (Green and Sambrook 2017) with minor modifications. Five g of sample was mixed with $10 \mathrm{ml}$ of lysis buffer, incorporating the SDS as well, (0.1 M Tris- $\mathrm{HCl} \mathrm{pH}$ 7.5, $0.05 \mathrm{M}$ EDTA pH 8, $1.25 \%$ SDS) and 10 $\mu \mathrm{l}$ Rase A $(100 \mathrm{mg} / \mathrm{ml})$. Then sediment was vortexed for $15 \mathrm{~s}$ and incubated at $37^{\circ} \mathrm{C}$ for $1 \mathrm{~h}$ in a hybridization oven. $100 \mu \mathrm{l}$ Proteinase $\mathrm{K}(20 \mathrm{mg} / \mathrm{ml})$ was added in a subsequent step and the mixture was incubated for $10 \mathrm{~min}$ at $70{ }^{\circ} \mathrm{C}$. Samples were extracted once with phenol/chloroform/isoamyl alcohol (ratio 25:24:1) and supernatants were extracted subsequently with chloroform/isoamyl alcohol (24:1). More stringent DNA precipitation conditions were applied with the addition of 10 $\mu \mathrm{g} / \mathrm{ml} \mathrm{LPA}$, and overnight incubation at $-20^{\circ} \mathrm{C}$ (Supplementary material).

All DNA extracts were suspended in $100 \mu 1$ of DNA/DNase-free water (ThermoFisher Scientific). Due to the inadequacy of DNA obtained from Method-1 given the $0.5 \mathrm{~g}$ input sediment weight, we scaled the extraction to $5 \mathrm{~g}$ starting weight prior to sequencing. Extracted DNA was thereafter stored at $-20{ }^{\circ} \mathrm{C}$ until further use. Due to the low DNA yields, it was necessary to use Qubit dsDNA HS kit (Invitrogen), a fluorescent DNA quantification method with high sensitivity. Quality assessment, with Nanodrop and DNA visualization on $0.8 \%$ agarose gel containing GelRed nucleic acid stain, was possible only for DNA extracted with method-4 and for DNA concentrations higher than $0.5 \mathrm{ng} / \mathrm{ul}$. All samples yielded sufficient DNA, i.e. $50 \mathrm{ng}$ (total input), for metagenomic sequencing and subsequent analyses. Additionally, a commercially-available microbial mock 
184 community (ZymoBIOMICS, Cat.No. D6300) was extracted using Method-4 and used for 185 subsequent sequencing.

186

187 DNA sequencing

$18850 \mathrm{ng}$ of DNA from all samples were subjected to random shotgun sequencing. The sequencing 189 libraries were prepared using the NEBNext Ultra II FS DNA Library Prep kit for Illumina (Cat.No. 190 E7805) using the protocol provided with the kit. The libraries were prepared considering 350 base 191 pairs (bp) average insert size. Qubit (Invitrogen) was used to quantify the prepared libraries while 192 their quality was assessed on a Bioanalyzer (Agilent). We used the NextSeq500 (Illumina) 193 instrument to perform the sequencing using 2x150 bp read length at the Luxembourg Centre for 194 Systems Biomedicine Sequencing Platform.

195

196 Genome reconstruction and metagenomic data processing

197 Paired sequences (i.e., forward and reverse) were processed using the Integrated Meta-omic 198 Pipeline (IMP) (Narayanasamy et al. 12/2016). The metagenomic workflow encompasses pre199 processing (read quality filtering and trimming), assembly, and genome reconstruction in a 200 reproducible manner. The adapter sequences were trimmed in the pre-processing step including

201 the removal of human reads. Thereafter, de novo assembly was performed using the MEGAHIT 202 (version 2.0) assembler (D. Li et al. 2015). Default IMP parameters were retained for all samples. 203 Subsequently, we used MetaBAT2 (Kang et al. 2019) and MaxBin2 (Wu, Simmons, and Singer 2016) for binning in addition to an in-house binning methodology previously described (HeintzBuschart et al. 2017). The latter method initially ignores the ribosomal RNA sequences in kmer profiles based on VizBin embedding clusters (Laczny et al. 2015). In this context, VizBin utilises

207 density-based non-hierarchical clustering algorithms and depth of coverage for genome 208 reconstructions. Subsequently we obtained a non-redundant set of metagenome-assembled genomes (MAGs) using DASTool (Sieber et al. 2018) with a score threshold of 0.7 for downstream analyses. The abundance of MAGs in each sample was determined by mapping the reads to the reconstructed genomes using BWA-MEM (H. Li 2013), taking the average coverage across all

212 contigs. Diversity measures from metagenomic sequencing were assessed by determining the 213 abundance-weighted average coverage of all the reads to identify the number of non-redundant 214 read sets (Rodriguez-R and Konstantinidis 2014). 
216 Taxonomic classification for metagenomic operational taxonomic units

217 We used the trimmed and pre-processed reads from the IMP workflow to determine the microbial 218 abundance and taxonomic profiles based on the mOTU (v2) tool (Milanese et al. 2019). Based on 219 the updated marker genes in the mOTU2 database including those from the TARA Oceans Study 220 (Sunagawa et al. 2015) and recently generated MAGs (Tully, Graham, and Heidelberg 2018), 221 taxonomic profiling was performed on our sequence datasets. We used a minimum alignment 222 length of $140 \mathrm{bp}$ to determine the relative abundances of the mOTUs, including the normalisation 223 of read counts to the gene length, also accounting for the base coverage of the genes. Additionally, 224 we used CheckM (Parks et al. 2015) to assess completeness and contamination. Subsequently, 225 taxonomy for MAGs recovered after the redundancy analyses from DASTool was determined 226 using the GTDB (Genome Taxonomy Database) toolkit (gtdb-tk) (Parks et al. 2018).

227

228 Data analysis

229 All figures for the DNA concentrations, library preparation, assembly metrics and supplementary 230 figures were generated using GraphPad Prism (v8.3.0). Taxonomical assessment and diversity 231 measures were created using version 3.6 of the R statistical software package (Team 2013). 232 DESeq2 (Love, Huber, and Anders 2014) with FDR-adjustments for multiple testing were used to 233 assess significant differences in the MAG abundances. The genomic cluster figure for the mock 234 community was obtained as an output from the IMP metagenomic pipeline.

\section{Results}

\section{Phenol-chloroform-based extraction method results in higher DNA yields}

238 To ensure native sequencing, by minimizing the number of PCR (polymerase chain reaction) 239 cycles within the library preparation protocols, we tested four protocols for biomolecular 240 extraction, with an aim of acquiring large quantities ( $>50 \mathrm{ng}$ ) DNA from glacier-fed stream benthic 241 sediments. The four methods tested were selected because of their wide applicability on related 242 environmental samples (Method-1 \& -2) (Griffiths et al. 2000; Lever et al. 2015; Tatti et al. 2016) 243 and their improved chances of higher yields (Method-3; Qiagen communication). Since method-4 244 is considered the gold standard of DNA extraction in biomedical sciences (Dairawan and Shetty 245 2020) and bacterial cultures (Green and Sambrook 2017), it was included in our study. The four 
246 protocols are largely based on the same principles, viz. sample preparation, cell lysis, purification, 247 precipitation and washing (Table 1). From preliminary tests, it became apparent that a small-scale 248 approach (Method-1; $0.5 \mathrm{~g}$ input sediment) did not yield sufficient amounts of DNA for 249 metagenomics due to, on average, limited microbial biomass in the samples. Thus, all protocols 250 (aside from Qiagen's - already produced for maxi scale) were scaled up to $5 \mathrm{~g}$ of input sediment 251 and a co-precipitant, like linear polyacrylamide, was included in all precipitation steps. This was

252 253 254

255

256

257

258

259

260

261

262

263

264

265

266

267

268

269

270

271

272

273

274

275

276 essential for the quantitative recovery of the small amounts of extracted DNA from high solution volumes $(6-10 \mathrm{ml})$.

Overall, we found that extractions using the commercial kit from Qiagen (method-3) yielded increased total DNA as compared to a commonly used protocol (method-1; Fig. 1A). Furthermore, method-3 was similar in terms of DNA yield when compared to a generalized protocol (method2) previously proposed (Lever et al. 2015) (Fig. 1B). On the other hand, the phenol-chloroform based extraction protocol (method-4) was tested against both methods 2 and 3, using sediment samples collected from the three different glacier floodplain streams (CBS, FEU, FED) from Switzerland. Method-1 was omitted from these tests due to insufficient DNA concentrations in the preliminary extractions. We found that for all three GFS, the phenol-chloroform extraction yielded the highest DNA concentrations. In some cases, and notably samples with low cell abundance, we even obtained one order of magnitude more DNA (Fig. 1C).

Quality assessment of these DNA extracts with Nanodrop showed $\mathrm{OD}_{260 / 280}$ ratios between $\sim 1.4$ and 1.6. Agarose gel electrophoresis revealed a high-molecular weight band with no apparent shearing, smearing or residual RNA, indicative of high-quality DNA (Fig. 2A). A secondary effect appearing in certain samples, but without any perceived consequences in the quality of extracted DNA whatsoever, was the development of a pink-red color of varying intensities with the addition of phenol:chloroform:isoamyl alcohol (Fig. 2B). This was $\mathrm{pH}$ dependent since samples were decolorized with the addition of sodium acetate $\mathrm{pH} 5.2$ in the precipitation step. This could possibly be due to a ferric-chloride-phenol compound formed when chloride and phenol constituents of the protocol interact reversibly with $\mathrm{Fe}^{+3}$ ions contained in certain samples depending on local geology (Banerjee and Haldar 1950). Similar coloration has been previously reported (Lever et al. 2015). 


\section{Extraction method affects library preparation efficiency}

279 The DNA extractions based on method-3 and using phenol-chloroform methods were subsequently 280 subjected to library preparation for high-throughput whole genome shotgun sequencing. Despite 281 similar quality of DNA across both methods $\left(\sim 1.4-1.6 \mathrm{OD}_{260 / 280}\right)$, library preparation using the 282 modified commercial kit did not yield any successful libraries (Fig. 3). To assess if any impurities 283 or inhibitors hampered library preparation we tested two clean-up methods for the DNA extracted from the commercial kit: 1) ethanol precipitation and 2) magnetic-bead based clean-up. For magnetic bead clean-up the SPRIselect beads (Beckman Coulter, 23318) were used according to

286

287

288

289

290

291

292

293

294

295

296

297

298

299

300

301

302

303

304

305

306 the manufacturer's protocol. We found that the magnetic-bead method leads to a complete loss of sample (i.e., undetectable DNA quantity via Qubit analyses) during the process, especially if starting with a low input DNA concentration. Although we lost six out of twelve samples using the magnetic-bead clean-up, we achieved 100\% efficiency as indicated by a concentration of greater than $0.5 \mathrm{ng} / \mathrm{ul}$ after library preparation quantified by Qubit, with the remaining six samples. On the other hand, $\sim 20 \%$ of the samples cleaned via ethanol precipitation failed library preparation. Contradictory to these methods, DNA extracted using the phenol-chloroform based method (method-4) yielded 100\% efficiency in terms of library preparation without any additional cleanup (Fig. 3). Additionally, we found that the distribution of the total yield after library preparation using the phenol-chloroform method was more uniform across samples compared to the other methods (Fig. 3).

\section{Whole genome shotgun assembly unaffected by extraction methods}

Extraction methods for whole genome shotgun sequencing may affect the sequencing itself, including the quality and assembly of the reads downstream. To assess this, we used the libraries prepared as described above (Fig. 3), and performed whole genome shotgun sequencing on an Illumina NextSeq500. The average quality across all three methods based on short-read sequencing was Q30 after trimming the leading and trailing sequences (described in Methods). We assessed several assembly metrics including the average length of contigs (N50), largest alignment, total aligned length and coverage. We did not find any significant differences among any of these measures across all three methods (Fig. 4A-C, 4E). Using a diversity index metric, we however 
307 found a more uniform distribution across all samples prepared using method-4, albeit no 308 significant differences to the commercial kit-based extraction and library preparation (Fig. 4D).

309

310 Extraction methods influence metagenomic profiles

311 It is well established that extraction methods (Wagner Mackenzie, Waite, and Taylor 2015) and

312 library preparation (Bowers et al. 2015) protocols affect the taxonomic profiles and genomes

313 recovered after high-throughput sequencing. We determined if the preparation methods affected

314 the overall diversity of taxa recovered and found that phenol-chloroform and the magnetic-bead 315 clean-up methods demonstrated similar levels of diversity (Shannon) as compared to samples 316 precipitated using ethanol (Fig. 5A). Overall, the community profiles of the ethanol precipitation317 based method were highly diverse (Fig. 5B). Interestingly, the genomes recovered and their 318 abundances were similar in the phenol-chloroform and magnetic-bead methods as well (Fig. 5C). 319 However, we observed a significant increase ( $p<0.001$, FDR-adjusted $p$-value) in the abundance 320 of a Ralstonia genome when prepared with the ethanol precipitation protocol (Supplementary 321 fig.1). Additionally, we found that the number of genomes recovered using the phenol-chloroform 322 was more consistent with previously reported 16S rRNA gene sequencing profiles for GFS from 323 Austria (Wilhelm et al. 2013; Besemer et al. 2012; Wilhelm et al. 2014). Simultaneously, we used 324 an approach to identify metagenomic operational taxonomic units (mOTUs) and found that the 325 phenol-chloroform and magnetic-bead methods showed similar profiles of mOTUs compared to 326 that of ethanol precipitation (Fig. 5D).

327

\section{Efficiency of phenol-chloroform extraction on a mock community including eukaryotes}

329 To determine whether the phenol-chloroform extraction method is biased against eukaryotes, we 330 used a commercially-available mock community (ZymoBIOMICS Microbial Community Standard \#D6300) to assess bias and errors. After sequencing, we recovered high quality ( $>90 \%$ completion, $<5 \%$ contamination) bacterial genomes (Fig. 6A). Additionally, the abundance of the microbial genomes, including one of the eukaryotes - Saccharomyces cerevisiae, were similar to the expected levels in the mock community (Fig. 6B). On the other hand, the protocol enabled the identification and partial recovery of the Cryptococcus neoformans genome, albeit at lower levels

337 lysis and subsequent extraction. 


\section{Discussion}

340 Improved omic techniques not limited to metagenomics are robust methods for analyzing nucleic 341 acids and the characterisation of microbial communities in various environments (Jansson et al.

342 2012). One way of understanding the impacts of global climate change on GFS includes the 343 establishment of their census of microbial life (Milner et al. 2017). However, methods designed 344 for the extraction of biomolecules including DNA have not been validated for GFS sediments. 345 Although previous glacier-fed streams studies successfully used extracted DNA for 16S rRNA 346 amplicon sequencing (Ren et al. 2017; Ren, Gao, and Elser 2017; Vardhan Reddy et al. 2009; 347 Wilhelm et al. 2013) the input DNA concentration requirements are considerably higher for whole 348 genome shotgun sequencing. In order to pursue a deeper characterisation of the microbial 349 communities within the GFS sediments, increased concentrations of DNA may additionally 350 alleviate PCR biases (Brooks et al. 2015; Kim and Bae 2011). Also, as previously highlighted, 351 several methods exist for extractions from a wide variety of environmental samples, but not for 352 GFS sediments. Here, we systematically tested the utility of four extraction protocols to identify a 353 ubiquitous methodology. We found that a phenol-chloroform based extraction protocol can be

354 355 356

357 358 359 360 361 362 363 364 365 366 367 368 used for samples across geographical separations, differences in bedrock, and samples collected at various distances from the glacier.

Glassing et al. demonstrated that inherent DNA contamination may influence microbiota interpretation in low biomass samples (Glassing et al. 2016). Additionally, it is known that certain compounds - polysaccharides, humic acids, may affect PCR reactions (Rådström et al. 2004), requiring the need for additional DNA clean-up. It has been established that DNA losses occur during the purification steps (Roose-Amsaleg, Garnier-Sillam, and Harry 2001), including when using commercial column methods (Howeler, Ghiorse, and Walker 2003; Lloyd et al. 2013), and phenol-chloroform (Ogram, Sayler, and Barkay 1987). Interestingly, we found similar losses when using the magnetic bead clean-up, whereas the ethanol precipitation method was inefficient compared to the phenol-chloroform protocol. Though the kit-based methods are more convenient and safer than phenol-chloroform extractions (Tesena et al. 2017), access to reagents and costs may be a considerable factor. On the other hand, isolation of the aqueous phase from phenolchloroform can be user-dependent potentially affecting reproducibility, while kits have been 
369 shown to be more consistent across samples (Claassen et al. 2013). Another key feature of our 370 findings was the potential for the kit-based methods to influence the efficiency of genome 371 reconstruction and variability in the taxonomic profiles that were recovered. While this has been 372 reported previously (Wagner Mackenzie, Waite, and Taylor 2015; Carrigg et al. 2007), we found 373 considerable variability when compared to the phenol-chloroform. This is plausible due to the 374 incomplete dissolution of DNA in buffers, especially when using methods involving charged 375 minerals (Vorhies and Gaines 2009; Barton et al. 2006; Vishnivetskaya et al. 2014), which may 376 additionally affect DNA stability.

377

\section{Conclusions}

379 The utility of extraction methods extends beyond the process itself, impacting downstream 380 applications such as whole genome shotgun sequencing. Our study shows that phenol-chloroform 381 may be an under-appreciated yet powerful method for isolating nucleic acids from glacier-fed

382

383

384

385

386

387

388

389

390

391

392

393

394

395

396

397

398

399 stream sediments. While additional steps may be required towards extraction of other biomolecules such as RNA, proteins and metabolites, minor modifications may be sufficient (Toni et al. 2018). Moreover, we report for the first time a systematic assessment of biomolecular extraction methods

for GFS sediments. Our findings though fundamental and previously unexplored, may lay the foundations for future in-depth characterisation of GFS microbial communities.

\section{Data Availability}

The sequencing data generated during the current study are available from NCBI under the BioProject accession number PRJNA624048. A reporting summary for the uploaded data has been included as a metadata file at the accession listed ID. All extraction protocols including the modified commercial methods are available in the Supplementary Materials.

\section{Acknowledgments}

We are grateful to Laura de Nies, Camille-Martin Gallausiaux, Jean-Pierre Trezzi, Cedric Laczny, Audrey Frachet, Lea Grandmougin, Annegrat Daujemont, Laura Lebrun (LCSB) for discussions and laboratory support. The University of Luxembourg Sequencing Platform and HPC facilities were highly instrumental for the in-silico analyses. The present work was supported by NOMIS Foundation and the Swiss National Science Foundation (CRSII5_180241) to Tom J. Battin. 
400

\section{Contributions}

402 P.P. performed the biomolecular extractions, including the validation of methods 1-3 alongside 403 quality analyses and quantification. S.B.B. curated and validated the phenol-chloroform extraction 404 method and whole genome shotgun sequencing analyses. T.B. and H.P. collected the glacier-fed 405 stream samples for the experiments. S.F. did the DNA extractions, quantification and 406 qualifications alongside P.P. R.H. handled the library preparation for all samples and the 407 subsequent sequencing. P.W. contributed significantly to the development of method-1 in the 408 manuscript. S.B.B., P.P., S.F., H.P., P.W., and T.J.B. conceived and formulated the experiments. 409 S.B.B. and P.P. developed the manuscript with equal contributions from all authors.

411 Ethical declarations

412 Conflicts of interest

413 The authors do not have any competing interests.

414

415 Figure legends

416 Figure 1. Total DNA concentrations using different extraction protocols

417 Boxplots represent the total amount of DNA (ng) extracted from $5 \mathrm{~g}$ of sediment when comparing 418 (A) method-1 versus the modified-commercial kit-based method-3 and (B) method-2 versus 419 method-3. (C) Boxplots of the DNA quantities isolated from three glacial floodplains (CBS 420 Corbassière, FEU - Val Ferret up site, FED - Val Ferret down site), using method -2, -3 and -4. 421 Method-1: CTAB buffer lysis (Griffiths et al. 2000), Method-2: Modular DNA extraction (Lever 422 et al. 2015), Method-3: Qiagen PowerMax Soil DNA extraction kit, Method-4: Chemical and 423 enzymatic extraction. Significance was tested using a Two-Way ANOVA with Student-Neuman 424 Keul's post-hoc analyses. $* * p<0.01, * * * p<0.001$

425

426 Figure 2. Characteristics of DNA extracted with method-4

427 (A) Agarose gel electrophoresis of DNA extracted with mild vortexing of sediments and 428 incubation in lysis buffer, proteinase $\mathrm{K}$ treatment and phenol-chloroform extraction. Lane 1: 429 GeneRuler 1 kb DNA ladder; lanes 2-4: CBS, FED, FEU respectively. (B) Pink-red supernatants 430 developed during phenol:chloroform extraction step. 
431

432

433 The efficiency or success percentage for prepared libraries based on the individual methods is 434 indicated in the table. Boxplots represent concentrations of the prepared libraries.

435

436

437

438

439

440

441

442

443

444

445

446

447

448

449

450

451

452

453

454

455

456

457

458

459

460

461

Figure 4. Estimate of assembly metrics following extraction

Barplots demonstrate the (A) N50 for the sequence assemblies, (B) length of the longest aligned sequence, (C) the total aligned length. Bars indicate standard deviation from the mean. (D) Boxplot showing the nonpareil diversity index across the three groups. (E) Percentage of coverage of the assembled sequences by read-mapping is depicted.

Figure 5. Diversity and taxonomic profiles of the metagenomic sequencing

(A) Boxplot showing the Shannon diversity index for the taxonomic profiling for the three groups. Significance was tested using a One-way ANOVA with Student-Neuman Keul's post-hoc analysis. $* * * p$-value $<0.001, * * * * p$-value $<0.0001$. (B) Principal component analyses generated using BrayCurtis dissimilarity matrix depicts similarities or lack thereof between the three groups. (C) Abundances of the reconstructed genomes are depicted for method-3 + EtOH, method-3 + magnetic bead clean-up and method-4 extraction. (D) Heatmap demonstrating the mOTUs for the three methods is depicted. The hierarchical clustering for the heatmap was generated using Ward's clustering algorithm.

Figure 6. Evaluation of phenol-chloroform extraction using a mock community

(A) Scatterplot depicts the clusters of contigs representative of the reconstructed genomes after processing the mock community using the IMP meta-omics pipeline. The taxonomic identity is displayed next to the respective clusters. (B) Barplots indicate the relative abundance of the individual genomes recovered from the mock community sequencing after extraction with the phenol-chloroform method. The upper (black) line represents the expected abundance (12\%) of the prokaryotes, while the lower (red) line indicates the expected abundance $(2 \%)$ of the eukaryotes.

\section{Supplementary figure 1 .}


462

463

464

465

466

467

468

469

470

471

472

473

474

475

476

477

478

479

480

481

482

483

484

485

486

487

488

489

490

491

492

493

494

495

496

497

498

499

500

501

502

503

504

505

506

507

Supplementary figure 1. Relative abundance of Ralstonia sp. AU12-08

The abundance of the Ralstonia genome recovered from the samples when processed with method-

3 (EtOH and magnetic bead clean-up) and method-4. Significance was tested using One-Way

ANOVA with Student-Neuman Keul's post-hoc analyses. $* * * p<0.001, * * * * p<0.0001$

\section{References}

Banerjee, S., and B. C. Haldar. 1950. "Constitution of Ferri-Phenol Complex in Solution." Nature 165 (4208): 1012.

Barton, H. A., N. M. Taylor, B. R. Lubbers, and A. C. Pemberton. 2006. "DNA Extraction from Low-Biomass Carbonate Rock: An Improved Method with Reduced Contamination and the Low-Biomass Contaminant Database." Journal of Microbiological Methods 66 (1): 21-31.

Besemer, Katharina, Hannes Peter, Jürg B. Logue, Silke Langenheder, Eva S. Lindström, Lars J. Tranvik, and Tom J. Battin. 2012. "Unraveling Assembly of Stream Biofilm Communities." The ISME Journal 6 (8): 1459-68.

Bogen, Jim. 1988. "Glacial Sediment Production and Development of Hydro-Electric Power in Glacierized Areas” 13 (January). https://doi.org/10.1017/S0260305500007539.

Bowers, Robert M., Alicia Clum, Hope Tice, Joanne Lim, Kanwar Singh, Doina Ciobanu, Chew Yee Ngan, Jan-Fang Cheng, Susannah G. Tringe, and Tanja Woyke. 2015. "Impact of Library Preparation Protocols and Template Quantity on the Metagenomic Reconstruction of a Mock Microbial Community." BMC Genomics 16 (October): 856.

Brooks, J. Paul, David J. Edwards, Michael D. Harwich Jr, Maria C. Rivera, Jennifer M. Fettweis, Myrna G. Serrano, Robert A. Reris, Nihar U Sheth, Bernice Huang, Phillippe Girerd, Vaginal Microbiome Consortium, Jerome F Strauss III, Kimberly K Jefferson and Gregory A Buck. 2015. "The Truth about Metagenomics: Quantifying and Counteracting Bias in 16S rRNA Studies." BMC Microbiology 15 (March): 66.

Carrigg, Cora, Olivia Rice, Siobhán Kavanagh, Gavin Collins, and Vincent O'Flaherty. 2007. "DNA Extraction Method Affects Microbial Community Profiles from Soils and Sediment." Applied Microbiology and Biotechnology 77 (4): 955-64.

Chafee, Meghan, Loïs Maignien, and Sheri L. Simmons. 2015. "The Effects of Variable Sample Biomass on Comparative Metagenomics." Environmental Microbiology 17 (7): 2239-53.

Chanudet, Vincent, and Montserrat Filella. 2008. "Size and Composition of Inorganic Colloids in a Peri-Alpine, Glacial Flour-Rich Lake." Geochimica et Cosmochimica Acta 72 (5): 146679.

Claassen, Shantelle, Elloise du Toit, Mamadou Kaba, Clinton Moodley, Heather J. Zar, and Mark P. Nicol. 2013. "A Comparison of the Efficiency of Five Different Commercial DNA Extraction Kits for Extraction of DNA from Faecal Samples." Journal of Microbiological Methods 94 (2): 103-10.

Dahm, Ralf. 2008. "Discovering DNA: Friedrich Miescher and the Early Years of Nucleic Acid Research." Human Genetics 122 (6): 565-81.

Dairawan, Mariyam, and Preetha J. Shetty. 2020. "The Evolution of DNA Extraction Methods." American Journal of Biomedical Science \& Research 8 (1). https://doi.org/10.34297/AJBSR.2020.08.001234.

Dancer, S. J., P. Shears, and D. J. Platt. 1997. "Isolation and Characterization of Coliforms from Glacial Ice and Water in Canada's High Arctic.” Journal of Applied Microbiology 82 (5): 597-609.

Glassing, Angela, Scot E. Dowd, Susan Galandiuk, Brian Davis, and Rodrick J. Chiodini. 2016.

Peer] reviewing PDF | (2020:05:48507:1:2:NEW 12 Aug 2020) 
508

509

510

511

512

513

514

515

516

517

518

519

520

521

522

523

524

525

526

527

528

529

530

531

532

533

534

535

536

537

538

539

540

541

542

543

544

545

546

547

548

549

550

551

552

553

554

555

556

557

558

"Inherent Bacterial DNA Contamination of Extraction and Sequencing Reagents May Affect Interpretation of Microbiota in Low Bacterial Biomass Samples." Gut Pathogens 8 (May): 24.

Godone, Danilo. 2017. Glacier Evolution in a Changing World. BoD - Books on Demand.

Green, Michael R., and Joseph Sambrook. 2017. "Isolating DNA from Gram-Negative Bacteria." Cold Spring Harbor Protocols 2017 (1). https://doi.org/10.1101/pdb.prot093369.

Griffiths, R. I., A. S. Whiteley, A. G. O'Donnell, and M. J. Bailey. 2000. "Rapid Method for Coextraction of DNA and RNA from Natural Environments for Analysis of Ribosomal DNAand rRNA-Based Microbial Community Composition." Applied and Environmental Microbiology 66 (12): 5488-91.

Grossman, Nina T., and Arturo Casadevall. 2017. "Physiological Differences in Cryptococcus Neoformans Strains In Vitro versus In Vivo and Their Effects on Antifungal Susceptibility." Antimicrobial Agents and Chemotherapy 61 (3). https://doi.org/10.1128/AAC.02108-16. Hale, Lauren, and David Crowley. 2015. "DNA Extraction Methodology for Biochar-Amended Sand and Clay." Biology and Fertility of Soils 51 (6): 733-38.

Heintz-Buschart, Anna, Patrick May, Cédric C. Laczny, Laura A. Lebrun, Camille Bellora, Abhimanyu Krishna, Linda Wampach, Jochen G Schneider, Angela Hogan, Carine de Beaufort, and Paul Wilmes. 2017. "Integrated Multi-Omics of the Human Gut Microbiome in a Case Study of Familial Type 1 Diabetes." Nature Microbiology 2 (1): 16180.

Hotaling, Scott, Eran Hood, and Trinity L. Hamilton. 2017. "Microbial Ecology of Mountain Glacier Ecosystems: Biodiversity, Ecological Connections and Implications of a Warming Climate." Environmental Microbiology 19 (8): 2935-48.

Howeler, Michael, William C. Ghiorse, and Larry P. Walker. 2003. "A Quantitative Analysis of DNA Extraction and Purification from Compost." Journal of Microbiological Methods 54 (1): 37-45.

Jansson, Janet K., and Kirsten S. Hofmockel. 2018. "The Soil Microbiome-from Metagenomics to Metaphenomics." Current Opinion in Microbiology, Environmental Microbiology * The New Microscopy, 43 (June): 162-68.

Jansson, Janet K., Josh D. Neufeld, Mary Ann Moran, and Jack A. Gilbert. 2012. "Omics for Understanding Microbial Functional Dynamics." Environmental Microbiology 14 (1): 1-3.

Kang, Dongwan D., Feng Li, Edward Kirton, Ashleigh Thomas, Rob Egan, Hong An, and Zhong Wang. 2019. "MetaBAT 2: An Adaptive Binning Algorithm for Robust and Efficient Genome Reconstruction from Metagenome Assemblies." PeerJ 7 (July): e7359.

Kebschull, Justus M., and Anthony M. Zador. 2015. "Sources of PCR-Induced Distortions in High-Throughput Sequencing Data Sets." Nucleic Acids Research 43 (21): e143.

Kim, Kyoung-Ho, and Jin-Woo Bae. 2011. "Amplification Methods Bias Metagenomic Libraries of Uncultured Single-Stranded and Double-Stranded DNA Viruses." Applied and Environmental Microbiology 77 (21): 7663-68.

Laczny, Cedric C., Tomasz Sternal, Valentin Plugaru, Piotr Gawron, Arash Atashpendar, Houry Hera Margossian, Sergio Coronado, Laurens van der Maaten, Nikos Vlassis, and Paul Wilmes. 2015. "VizBin - an Application for Reference-Independent Visualization and Human-Augmented Binning of Metagenomic Data." Microbiome 3 (1): 1.

Lever, Mark A., Andrea Torti, Philip Eickenbusch, Alexander B. Michaud, Tina Šantl-Temkiv, and Bo Barker Jørgensen. 2015. "A Modular Method for the Extraction of DNA and RNA, and the Separation of DNA Pools from Diverse Environmental Sample Types." Frontiers in Microbiology 6 (May): 476.

Li, Dinghua, Chi-Man Liu, Ruibang Luo, Kunihiko Sadakane, and Tak-Wah Lam. 2015. "MEGAHIT: An Ultra-Fast Single-Node Solution for Large and Complex Metagenomics Assembly via Succinct de Bruijn Graph." Bioinformatics 31 (10): 1674-76.

Li, Heng. 2013. "Aligning Sequence Reads, Clone Sequences and Assembly Contigs with BWAMEM." arXiv [q-bio.GN]. arXiv. http://arxiv.org/abs/1303.3997.

PeerJ reviewing PDF | (2020:05:48507:1:2:NEW 12 Aug 2020) 
559

560

561

562

563

564

565

566

567

568

569

570

571

572

573

574

575

576

577

578

579

580

581

582

583

584

585

586

587

588

589

590

591

592

593

594

595

596

597

598

599

600

601

602

603

604

605

606

607

608

609
Lloyd, Karen G., Lars Schreiber, Dorthe G. Petersen, Kasper U. Kjeldsen, Mark A. Lever, Andrew D. Steen, Ramunas Stepanauskas, Michael Richter, Sara Kleindienst, Sabine Lennk, Andreas Schramm and Bo Barker Jorgenson. 2013. "Predominant Archaea in Marine Sediments Degrade Detrital Proteins." Nature 496 (7444): 215-18.

Love, Michael I., Wolfgang Huber, and Simon Anders. 2014. "Moderated Estimation of Fold Change and Dispersion for RNA-Seq Data with DESeq2." Genome Biology 15 (12). https://doi.org/10.1186/s13059-014-0550-8.

Milanese, Alessio, Daniel R. Mende, Lucas Paoli, Guillem Salazar, Hans-Joachim Ruscheweyh, Miguelangel Cuenca, Pascal Hingamp, Renato Alves, Paul I Costea, Luis Pedro Coelho, Thomas S. B. Schmidt, Alexandre Almeida, Alex L Mitchell, Robert D. Finn, Jaime HuertaCepas, Peer Bork, Georg Zeller and Shinichi Sunagawa. 2019. "Microbial Abundance, Activity and Population Genomic Profiling with mOTUs2." Nature Communications 10 (1): 1014.

Miller, D. N., J. E. Bryant, E. L. Madsen, and W. C. Ghiorse. 1999. "Evaluation and Optimization of DNA Extraction and Purification Procedures for Soil and Sediment Samples." Applied and Environmental Microbiology 65 (11): 4715-24.

Milner, Alexander M., Kieran Khamis, Tom J. Battin, John E. Brittain, Nicholas E. Barrand, Leopold Füreder, Sophie Cauvy-Fraunié, Gísli Már Gíslason, Dean Jacobsen, David M Hannah, Andrew J Hodson, Eran Hood, Valeria Lencioni, Jón S Ólafsson, Christopher T Robinson, Martyn Tranter, Lee E Brown. 2017. "Glacier Shrinkage Driving Global Changes in Downstream Systems." Proceedings of the National Academy of Sciences of the United States of America 114 (37): 9770-78.

Mukhopadhyay, T., and J. A. Roth. 1993. "Silicone Lubricant Enhances Recovery of Nucleic Acids after Phenol-Chloroform Extraction." Nucleic Acids Research 21 (3): 781-82.

Narayanasamy, Shaman, Yohan Jarosz, Emilie E. L. Muller, Anna Heintz-Buschart, Malte Herold, Anne Kaysen, Cédric C. Laczny, Nicolás Pinel, Patrick May, and Paul Wilmes. 12/2016. "IMP: A Pipeline for Reproducible Reference-Independent Integrated Metagenomic and Metatranscriptomic Analyses." Genome Biology 17 (1): 260.

Nielsen, Jens, and Boyang Ji. 2015. "New Insight into the Gut Microbiome through Metagenomics." Advances in Genomics and Genetics. https://doi.org/10.2147/agg.s57215.

Ogram, Andrew, Gary S. Sayler, and Tamar Barkay. 1987. "The Extraction and Purification of Microbial DNA from Sediments." Journal of Microbiological Methods 7 (2): 57-66.

Parks, Donovan H., Maria Chuvochina, David W. Waite, Christian Rinke, Adam Skarshewski, Pierre-Alain Chaumeil, and Philip Hugenholtz. 2018. "A Proposal for a Standardized Bacterial Taxonomy Based on Genome Phylogeny." bioRxiv, January, 256800.

Parks, Donovan H., Michael Imelfort, Connor T. Skennerton, Philip Hugenholtz, and Gene W. Tyson. 2015. "CheckM: Assessing the Quality of Microbial Genomes Recovered from Isolates, Single Cells, and Metagenomes." Genome Research 25 (7): 1043-55.

Pei-Ying, Yan, Hou Shu-Gui, Chen Tuo, Zhang Shu-Hong, and Sun Wei-Jun. 2012. "Methods for Extraction of Microorganism DNA from Glacier Surface Snow." Sciences in Cold and Arid Regions 4 (6): 484.

Peng, Zonghui, Xiaolong Zhu, Zhijiao Wang, Xianting Yan, Guangbiao Wang, Meifang Tang, Awei Jiang, and Karsten Kristiansen. 2020. "Comparative Analysis of Sample Extraction and Library Construction for Shotgun Metagenomics." Bioinformatics and Biology Insights 14 (June): 1177932220915459.

Peter, Hannes, and Ruben Sommaruga. 2017. "Alpine Glacier-Fed Turbid Lakes Are Discontinuous Cold Polymictic rather than Dimictic." Inland Waters : Journal of the International Society of Limnology 7 (1): 45-54.

Porebski, Sue, L. Grant Bailey, and Bernard R. Baum. 1997. "Modification of a CTAB DNA Extraction Protocol for Plants Containing High Polysaccharide and Polyphenol Components." Plant Molecular Biology Reporter / ISPMB 15 (1): 8-15. 
610

611

612

613

614

615

616

617

618

619

620

621

622

623

624

625

626

627

628

629

630

631

632

633

634

635

636

637

638

639

640

641

642

643

644

645

646

647

648

649

650

651

652

653

654

655

656

657

658

659

660

Psifidi, Androniki, Chrysostomos I. Dovas, Georgios Bramis, Thomai Lazou, Claire L. Russel, Georgios Arsenos, and Georgios Banos. 2015. "Comparison of Eleven Methods for Genomic DNA Extraction Suitable for Large-Scale Whole-Genome Genotyping and LongTerm DNA Banking Using Blood Samples." PloS One 10 (1): e0115960.

Rådström, Peter, Rickard Knutsson, Petra Wolffs, Maria Lövenklev, and Charlotta Löfström. 2004. "Pre-PCR Processing: Strategies to Generate PCR-Compatible Samples." Molecular Biotechnology 26 (2): 133-46.

Ren, Ze, Hongkai Gao, and James J. Elser. 2017. "Longitudinal Variation of Microbial Communities in Benthic Biofilms and Association with Hydrological and Physicochemical Conditions in Glacier-Fed Streams." Freshwater Science 36 (3): 479-90.

Ren, Ze, Hongkai Gao, James J. Elser, and Qiudong Zhao. 2017. "Microbial Functional Genes Elucidate Environmental Drivers of Biofilm Metabolism in Glacier-Fed Streams." Scientific Reports 7 (October). https://doi.org/10.1038/s41598-017-13086-9.

Rodriguez-R, Luis M., and Konstantinos T. Konstantinidis. 2014. "Nonpareil: A RedundancyBased Approach to Assess the Level of Coverage in Metagenomic Datasets." Bioinformatics 30 (5): 629-35.

Roose-Amsaleg, C. L., E. Garnier-Sillam, and M. Harry. 2001. "Extraction and Purification of Microbial DNA from Soil and Sediment Samples." Applied Soil Ecology: A Section of Agriculture, Ecosystems \& Environment 18 (1): 47-60.

Roume, Hugo, Anna Heintz-Buschart, Emilie E. L. Muller, and Paul Wilmes. 2013. "Sequential Isolation of Metabolites, RNA, DNA, and Proteins from the Same Unique Sample." Methods in Enzymology 531: 219-36.

Sambrook, Joseph, and David W. Russell. 2006. "Purification of Nucleic Acids by Extraction with Phenol:chloroform." CSH Protocols 2006 (1). https://doi.org/10.1101/pdb.prot4455.

Sieber, Christian M. K., Alexander J. Probst, Allison Sharrar, Brian C. Thomas, Matthias Hess, Susannah G. Tringe, and Jillian F. Banfield. 2018. "Recovery of Genomes from Metagenomes via a Dereplication, Aggregation and Scoring Strategy." Nature Microbiology 3 (7): 836-43.

Smith, K., M. A. Diggle, and S. C. Clarke. 2003. "Comparison of Commercial DNA Extraction Kits for Extraction of Bacterial Genomic DNA from Whole-Blood Samples." Journal of Clinical Microbiology 41 (6): 2440-43.

Sunagawa, Shinichi, Luis Pedro Coelho, Samuel Chaffron, Jens Roat Kultima, Karine Labadie, Guillem Salazar, Bardya Djahanschiri, Georg Zeller, Daniel R Mende, Adriana Alberti, Francisco M Cornejo-Castillo, Paul I Costea, Corinne Cruaud, Francesco d'Ovidio, Stefan Engelen, Isabel Ferrera, Josep M Gasol, Lionel Guidi, Falk Hildebrand, Florian Kokoszka, Cyrille Lepoivre, Gipsi Lima-Mendez, Julie Poulain, Bonnie T Poulos, Marta Royo-Llonch, Hugo Sarmento, Sara Vieira-Silva, Céline Dimier, Marc Picheral, Sarah Searso, Stefanie Kandels-Lewis, Tara Oceans coordinators; Chris Bowler, Colomban de Vargas, Gabriel Gorsky, Nigel Grimsley, Pascal Hingamp, Daniele ludicone, Olivier Jaillon, Fabrice Not, Hiroyuki Ogata, Stephane Pesant, Sabrina Speich, Lars Stemmann, Matthew B Sullivan, Jean Weissenbach, Patrick Wincker, Eric Karsenti, Jeroen Raes, Silvia G Acinas, Peer Bork. 2015. "Ocean Plankton. Structure and Function of the Global Ocean Microbiome." Science 348 (6237): 1261359.

Tatti, Enrico, Boyd A. McKew, Corrine Whitby, and Cindy J. Smith. 2016. "Simultaneous DNARNA Extraction from Coastal Sediments and Quantification of 16S rRNA Genes and Transcripts by Real-Time PCR." Journal of Visualized Experiments: JoVE, no. 112 (June). https://doi.org/10.3791/54067.

Team, R. Core. 2013. "R: A Language and Environment for Statistical Computing."

Tesena, Parichart, Wasamon Korchunjit, Jane Taylor, and Tuempong Wongtawan. 2017. "Comparison of Commercial RNA Extraction Kits and qPCR Master Mixes for Studying Gene Expression in Small Biopsy Tissue Samples from the Equine Gastric Epithelium."

Peer) reviewing PDF | (2020:05:48507:1:2:NEW 12 Aug 2020) 
661

662

663

664

665

666

667

668

669

670

671

672

673

674

675

676

677

678

679

680

681

682

683

684

685

686

687

688

689

690

691

692

693

694

695

696

697

698

699

700
Journal of Equine Science 28 (4): 135-41.

Thomas, Torsten, Jack Gilbert, and Folker Meyer. 2012. "Metagenomics - a Guide from Sampling to Data Analysis." Microbial Informatics and Experimentation 2 (1): 3.

Toni, Lee S., Anastacia M. Garcia, Danielle A. Jeffrey, Xuan Jiang, Brian L. Stauffer, Shelley D. Miyamoto, and Carmen C. Sucharov. 2018. "Optimization of Phenol-Chloroform RNA Extraction." MethodsX 5 (May): 599-608.

Tully, Benjamin J., Elaina D. Graham, and John F. Heidelberg. 2018. "The Reconstruction of 2,631 Draft Metagenome-Assembled Genomes from the Global Oceans." Scientific Data 5 (January): 170203.

Vardhan Reddy, Puram Vishnu, Singireesu Soma Shiva Nageswara Rao, Mambatta Shankaranarayanan Pratibha, Buddhi Sailaja, Bakka Kavya, Ravoori Ruth Manorama, Shiv Mohan Singh, Tanuku Naga Radha Srinivas, and Sisinthy Shivaji. 2009. "Bacterial Diversity and Bioprospecting for Cold-Active Enzymes from Culturable Bacteria Associated with Sediment from a Melt Water Stream of Midtre Lovenbreen Glacier, an Arctic Glacier." Research in Microbiology 160 (8): 538-46.

Vishnivetskaya, Tatiana A., Alice C. Layton, Maggie C. Y. Lau, Archana Chauhan, Karen R. Cheng, Arthur J. Meyers, Jasity R. Murphy, Alexandra W Rogers, Geetha S Saarunya, Daniel E Williams, Susan M Pfiffner, John P Biggerstaff, Brandon T Stackhouse, Tommy J Phelps, Lyle Whyte, Gary S Sayler, and Tullis C Onstott. 2014. "Commercial DNA Extraction Kits Impact Observed Microbial Community Composition in Permafrost Samples." FEMS Microbiology Ecology 87 (1): 217-30.

Vorhies, John S., and Robert R. Gaines. 2009. "Microbial Dissolution of Clay Minerals as a Source of Iron and Silica in Marine Sediments." Nature Geoscience 2 (3): 221-25.

Wagner Mackenzie, Brett, David W. Waite, and Michael W. Taylor. 2015. "Evaluating Variation in Human Gut Microbiota Profiles due to DNA Extraction Method and Inter-Subject Differences." Frontiers in Microbiology 6 (February): 130.

Wilhelm, Linda, Katharina Besemer, Christina Fasching, Tim Urich, Gabriel A. Singer, Christopher Quince, and Tom J. Battin. 2014. "Rare but Active Taxa Contribute to Community Dynamics of Benthic Biofilms in Glacier-Fed Streams." Environmental Microbiology 16 (8): 2514-24.

Wilhelm, Linda, Gabriel A. Singer, Christina Fasching, Tom J. Battin, and Katharina Besemer. 2013. "Microbial Biodiversity in Glacier-Fed Streams." The ISME Journal 7 (8): 1651-60.

Wu, Yu-Wei, Blake A. Simmons, and Steven W. Singer. 2016. "MaxBin 2.0: An Automated Binning Algorithm to Recover Genomes from Multiple Metagenomic Datasets." Bioinformatics 32 (4): 605-7.

Xin, Zhanguo, and Junping Chen. 2012. "A High Throughput DNA Extraction Method with High Yield and Quality." Plant Methods. https://doi.org/10.1186/1746-4811-8-26.

Zhou, J., M. A. Bruns, and J. M. Tiedje. 1996. "DNA Recovery from Soils of Diverse Composition." Applied and Environmental Microbiology 62 (2): 316-22. 


\section{Figure 1}

Total DNA concentrations using different extraction protocols

Boxplots represent the total amount of DNA (ng) extracted from $5 \mathrm{~g}$ of sediment when comparing (A) method-1 versus the modified-commercial kit-based method-3 and (B) method-2 versus method-3. (C) Boxplots of the DNA quantities isolated from three glacial floodplains (CBS - Corbassière, FEU - Val Ferret up site, FED - Val Ferret down site), using method -2,-3 and -4. Method-1: CTAB buffer lysis (Griffiths et al. 2000), Method-2: Modular DNA extraction (Lever et al. 2015), Method-3: Qiagen PowerMax Soil DNA extraction kit, Method-4: Chemical and enzymatic extraction. Significance was tested using a Two-Way ANOVA with Student-Neuman Keul's post-hoc analyses. ${ }^{* *} p<0.01,{ }^{* *} p<0.001$ 
A

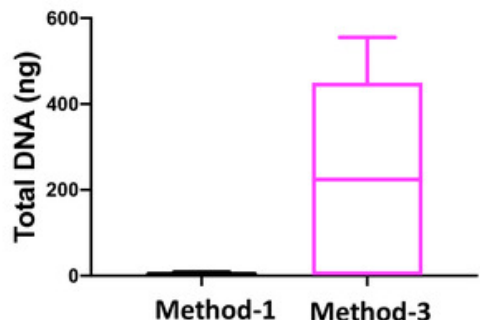

C

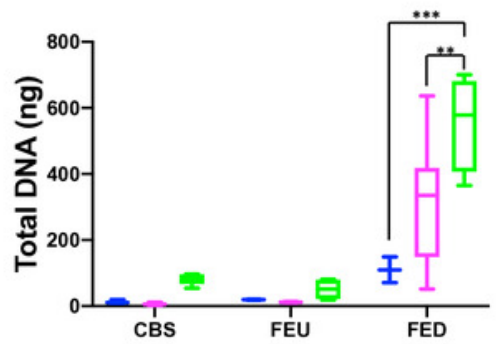

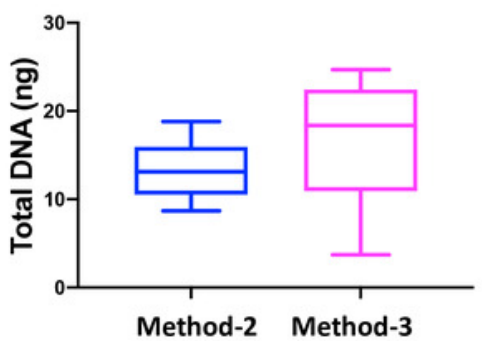

$\square$ Method-2

$\square$ Method-3

$\square$ Method-4 


\section{Figure 2}

Characteristics of DNA extracted with method-4

(A) Agarose gel electrophoresis of DNA extracted with mild vortexing of sediments and incubation in lysis buffer, proteinase $\mathrm{K}$ treatment and phenol-chloroform extraction. Lane 1: GeneRuler 1 kb DNA ladder; lanes 2-4: CBS, FED, FEU respectively. (B) Pink-red supernatants developed during phenol:chloroform extraction step.

A

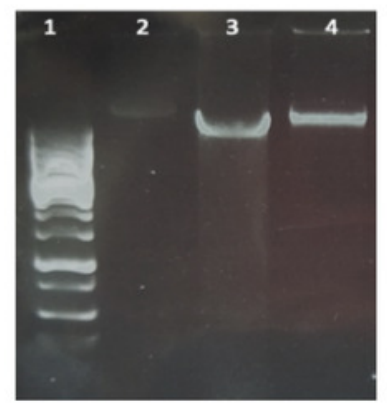

B

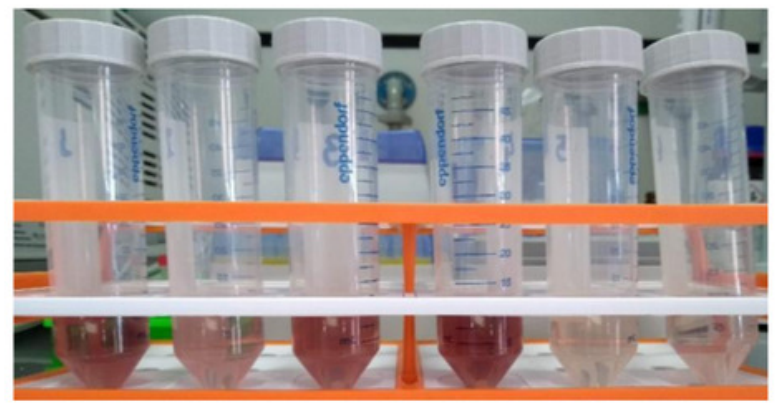


Figure 3

Figure 3. Library preparation efficiency

The efficiency or success percentage for prepared libraries based on the individual methods is indicated in the table. Boxplots represent concentrations of the prepared libraries.

A

\begin{tabular}{|l|c|}
\hline \multirow{2}{*}{ Extraction_method } & $\begin{array}{c}\text { Library Preparation } \\
\text { Efficiency }\end{array}$ \\
\cline { 2 - 2 } & Success (\%) \\
\hline Method-3 $(n=16)$ & 0 \\
\hline Method-3 + EtOH ( $=14)$ & 78.5 \\
\hline Method-3 + Magnetic_Beads $(n=12)$ & $100 *$ \\
\hline Method-4 $(n=12)$ & 100 \\
\hline
\end{tabular}

*Percentage based on 6 samples only, since 6 samples did not yield sufficient DNA after clean-up

$\mathrm{n}=$ number of samples
B

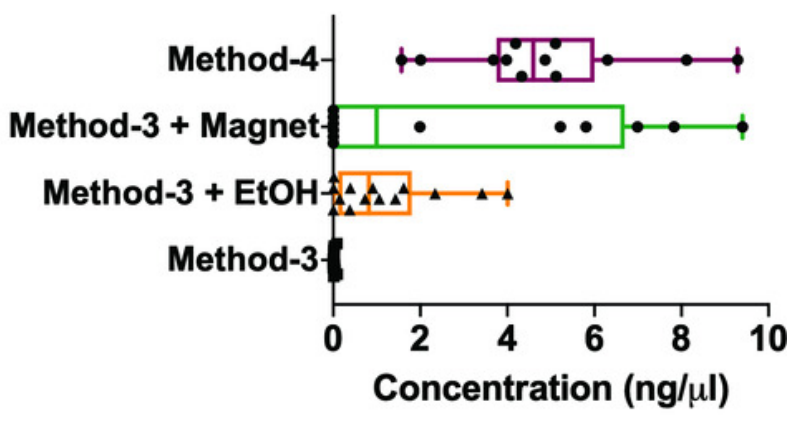


Figure 4

Estimate of assembly metrics following extraction

Barplots demonstrate the (A) N50 for the sequence assemblies, (B) length of the longest aligned sequence, $(\mathrm{C})$ the total aligned length. Bars indicate standard deviation from the mean. (D) Boxplot showing the nonpareil diversity index across the three groups. (E)

Percentage of coverage of the assembled sequences by read-mapping is depicted.

A

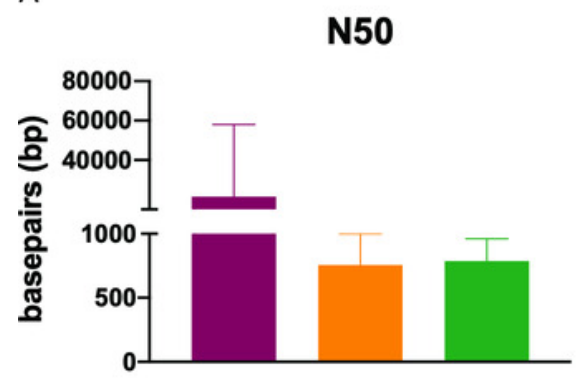

D

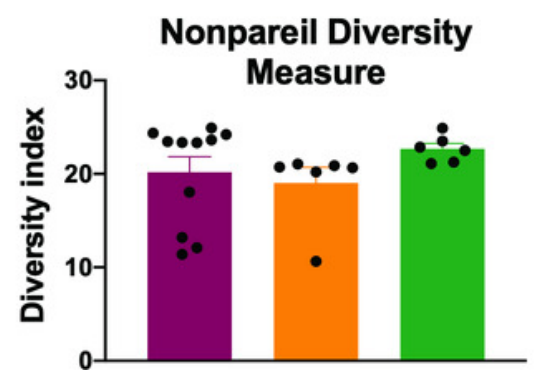

B

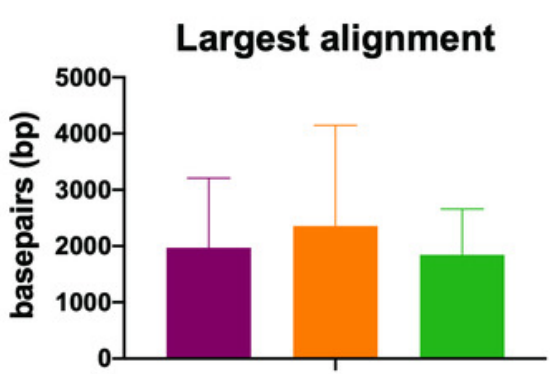

$E$

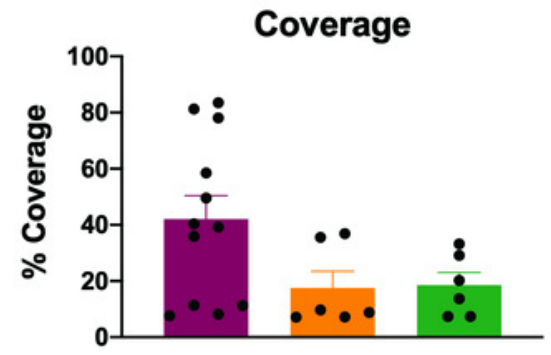

C

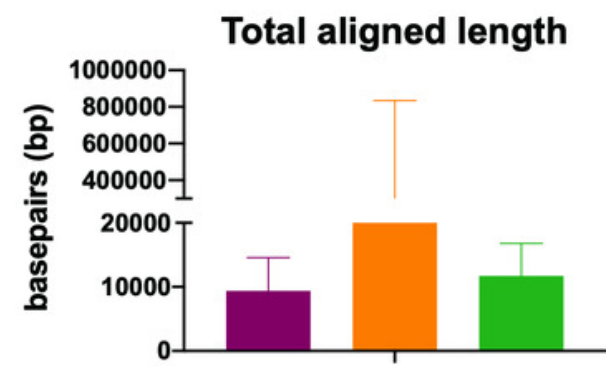

Extraction method

- Method-3 + EtOH

Method-3 + Magnet

Method-4 


\section{Figure 5}

Diversity and taxonomic profiles of the metagenomic sequencing

(A) Boxplot showing the Shannon diversity index for the taxonomic profiling for the three groups. Significance was tested using a One-way ANOVA with Student-Neuman Keul's posthoc analysis. ${ }^{* *} p$-value $<0.001,{ }^{* * *} p$-value $<0.0001$. (B) Principal component analyses generated using Bray-Curtis dissimilarity matrix depicts similarities or lack thereof between the three groups. (C) Abundances of the reconstructed genomes are depicted for method- $3+$ EtOH, method-3 + magnetic bead clean-up and method-4 extraction. (D) Heatmap demonstrating the mOTUs for the three methods is depicted. The hierarchical clustering for the heatmap was generated using Ward's clustering algorithm. 
A

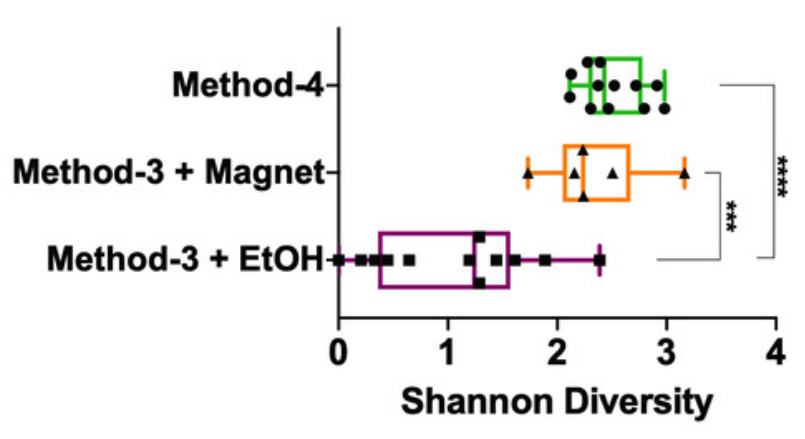

C

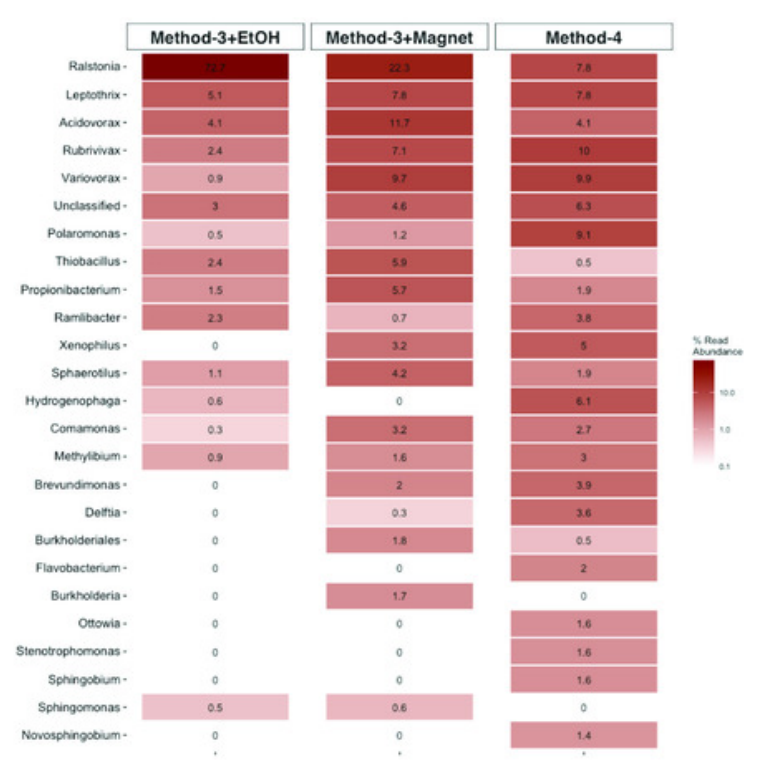

B

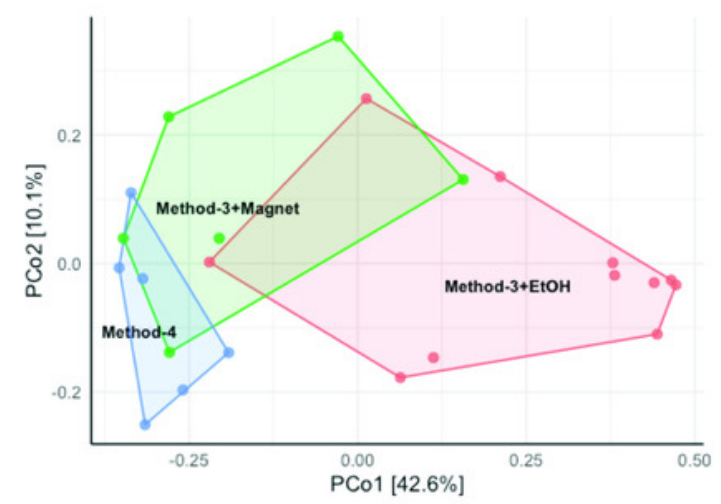

D

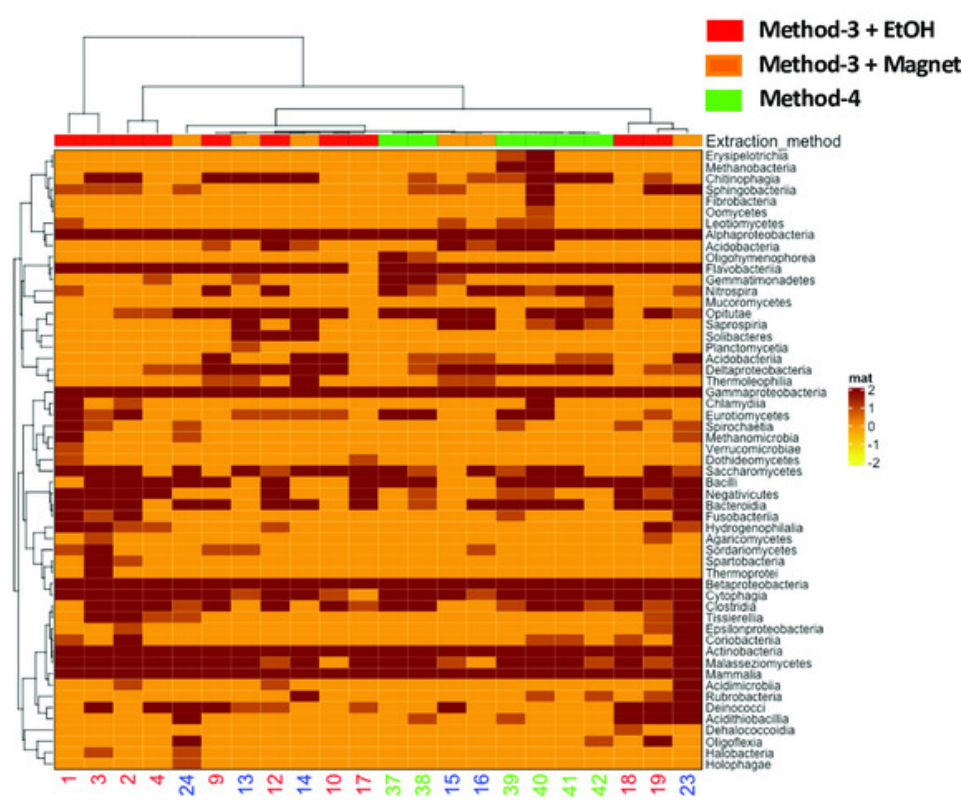




\section{Figure 6}

Evaluation of phenol-chloroform extraction using a mock community

(A) Scatterplot depicts the clusters of contigs representative of the reconstructed genomes after processing the mock community using the IMP meta-omics pipeline. The taxonomic identity is displayed next to the respective clusters. (B) Barplots indicate the relative abundance of the individual genomes recovered from the mock community sequencing after extraction with the phenol-chloroform method. The upper (black) line represents the expected abundance (12\%) of the prokaryotes, while the lower (red) line indicates the expected abundance (2\%) of the eukaryotes.

A

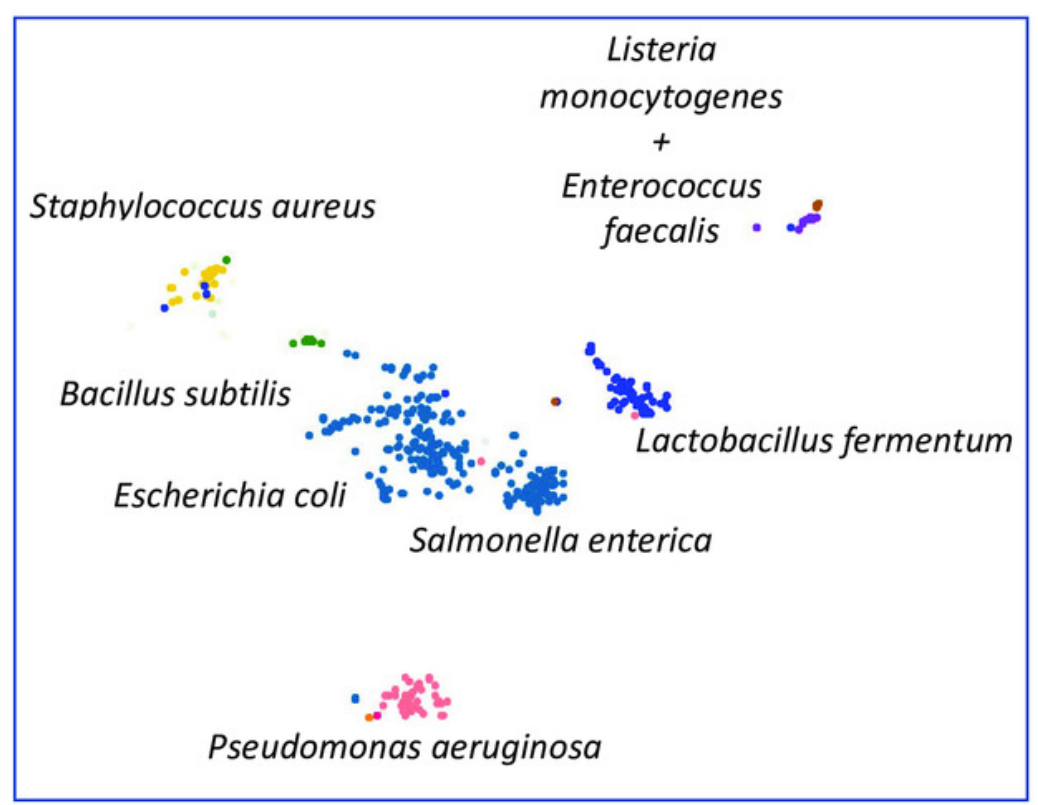

B

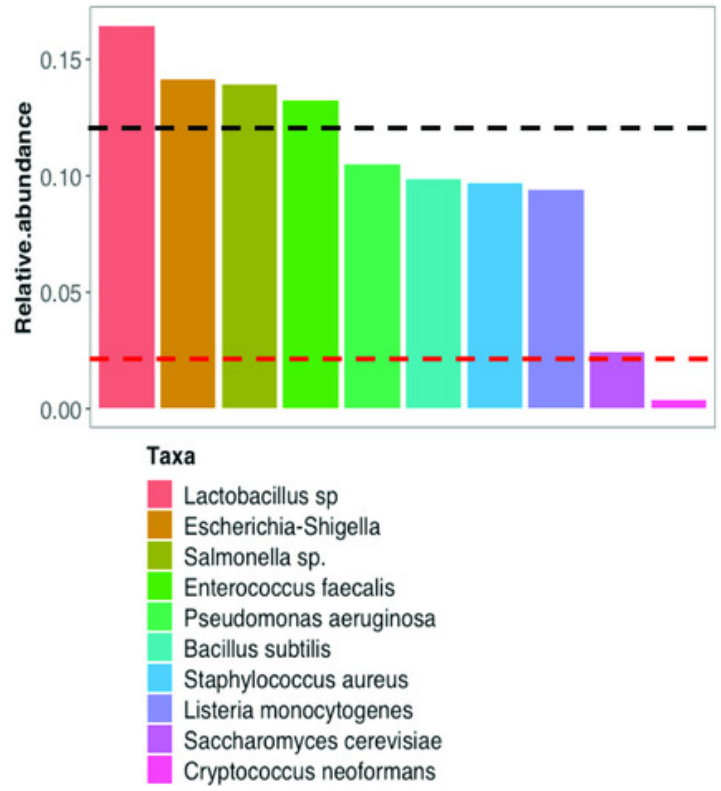




\section{Table $\mathbf{1}$ (on next page)}

Table 1: Key characteristics of the four selected methods

The table lists the key and specific characteristics of the four extraction methods tested, where $n$, is the total number of times each condition was tested on the material; RT: room temperature 


\begin{tabular}{|c|c|c|c|c|}
\hline & $\begin{array}{c}\text { Method-1 } \\
\quad(n=3)\end{array}$ & $\begin{array}{c}\text { Method-2 } \\
\quad(n=6)\end{array}$ & $\begin{array}{c}\text { Method-3 } \\
\quad(n=30)\end{array}$ & $\begin{array}{c}\text { Method-4 } \\
(n=14)\end{array}$ \\
\hline Sample prep & $\begin{array}{l}\text { Lysis matrix E tube } \\
\text { (beads diam 1.4, 0.1, } 4 \\
\mathrm{~mm} \text { ) }\end{array}$ & $\begin{array}{l}0.1 \mathrm{~mm} \text { Zirconium beads } \\
\text { dNTP solution }\end{array}$ & $\begin{array}{l}\text { PowerBead tubes } \\
\text { (carnet } 0.7 \mathrm{~mm} \text { ) }\end{array}$ & - \\
\hline Cell lysis & $\begin{array}{l}\text { Lysis buffer } \\
\text { CTAB/KaPO4, pH } 8 \\
\text { Phenol:Chloroform:Isoa } \\
\text { myl alcohol } \\
\text { Bead-beating }\end{array}$ & $\begin{array}{l}\text { Lysis buffer } \\
\text { GuHCl/EDTA/Triton } \\
\mathrm{X}-100, \mathrm{pH} 10 \\
\text { Mild vortex Incubation } \\
\text { at } 50^{\circ} \mathrm{C}\end{array}$ & $\begin{array}{l}\text { PowerBead Buffer+C1 } \\
\text { Phenol:Chloroform:Isoa } \\
\text { myl alcohol } \\
\text { Vortex (MO BIO vortex } \\
\text { adapter) }\end{array}$ & $\begin{array}{l}\text { Lysis Buffer } \\
\text { Tris-HCl, EDTA, SDS } \\
\text { pH } 8.0 \\
\text { Mild vortex } \\
\text { Incubation at } 37^{\circ} \mathrm{C} \\
\text { Proteinase } \mathrm{K} \text { addition } \\
\text { and incubation at } 70^{\circ} \mathrm{C}\end{array}$ \\
\hline Purification & $\begin{array}{l}\text { Chloroform:Isoamyl } \\
\text { alcohol (x1) }\end{array}$ & $\begin{array}{l}\text { Chloroform:Isoamyl } \\
\text { alcohol (x1) }\end{array}$ & $\begin{array}{l}\text { Inhibitor Removal } \\
\text { Technology }(\mathrm{C} 2, \mathrm{C} 3)\end{array}$ & $\begin{array}{l}\text { Phenol:Chloroform:Isoa } \\
\text { myl alcohol (x1) } \\
\text { Chloroform:Isoamyl } \\
\text { alcohol (x1) }\end{array}$ \\
\hline Precipitation & $\begin{array}{l}\text { Linear polyacrylamide } \\
\text { PEG-6000 } \\
\text { Ice }\end{array}$ & $\begin{array}{l}\text { Linear polyacrylamide } \\
\mathrm{NaCl} \\
\text { Ethanol } \\
\text { RT }\end{array}$ & $\begin{array}{l}\text { 1st: Column binding \& } \\
\text { cleaning } \\
\mathrm{C} 4, \mathrm{C} 4+\mathrm{EtOH}, \mathrm{C} 5 \text {, } \\
\text { EtOH } \\
\text { Elution: } 6 \mathrm{ml} \mathrm{EB} \\
\text { 2nd: Linear } \\
\text { polyacrylamide } \\
\mathrm{NaCl} \text {, Ethanol }\end{array}$ & $\begin{array}{l}\text { Linear Polyacrylamide } \\
\text { Sodium acetate } \\
\text { Isopropanol } \\
-20^{\circ} \mathrm{C}\end{array}$ \\
\hline
\end{tabular}

